\title{
Design and Development of Nonlinear Optical Microscope System: Simple Implementation with epi-Illumination Platform
}

\author{
Jiheun Ryu ${ }^{1}$, Jayul Kim¹, Hyunjun Kim¹, Hongki Yoo² and Daegab Gweon 1,a \\ ${ }^{1}$ Department of Mechanical Engineering, KAIST, Daejeon, South Korea \\ ${ }^{2}$ Department of Biomedical Engineering, Hanyang University, Seoul, South Korea
}

\begin{abstract}
During the research using fluorescence-tagged or auto-fluorescence molecules, meaningful information is often buried deep inside the tissue, not its surface. Therefore, especially in the field of biomedical imaging, acquiring optically sectioned images from deep inside the tissue is very important. As well know already, confocal laser scanning microscopy (the most well-known optical sectioning microscopy) gives axially-resolved fluorescence information using the physical background blocking component called pinhole. However, the axial range of imaging is practically limited due to such optical phenomena as the light scattered and absorbed in the tissue. However, nonlinear optical microscopy (e.g. Multiphoton microscopy, harmonic generation microscopy, coherent anti-Stokes Raman spectroscopy) realized by the development of ultrafast light sources has been used for visualizing various tissues, especially in vivo, because of their low sensitivity to the limitation caused by the scattering and the absorption of light. Although nonlinear optical microscopy gives deep tissue image, it is not easy for many researcher to build customized nonlinear system. Here, we introduce an easy and simple way designing and developing such nonlinear optical microscope with upright or inverted epi-illumination platform using commercial optical components only.
\end{abstract}

\section{Introduction}

Fluorescence microscopy utilizes fluorophores, manmade fluorescence dyes such as FITC, Cy5 and ICG, or auto-fluorescence molecules such as NADH, FAD, collagen and elastin for visualizing internal structure or cellular distribution of the tissue. Its major ability is that it gives us target-specified information from the various objects in the tissue [1]. However, unlike techniques which use physically sectioned samples of the tissue (e.g. Histology with Haematoxylin and eosin staining), intact tissue can be regarded as a finite stack of such physical sections. Therefore, we need some kind of technique to visualize one of the section in the axial stack.

Confocal microscopy, first invented by Marvin Minsky, is the most well-known technique for optical sectioning [2]. This means that it can give virtual slices of the tissue by physically getting rid of the light back scattered or emitted from other slices except for light from the target slice, and thus can give one the capability of three-dimensional reconstruction [3].

However, the usage of confocal microscopy is limited for its depth of penetration. In almost all of situations, the first question arises is for which section we should target to get meaningful information from the tissue. And the second question is that could the axial imaging range of our system covers that section. Similarly, in the 3D imaging applications, the key

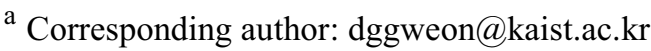

limitation converges to the axial imaging range of the system [4].

Two major factors causing practical limitation of the axial range of imaging are scattering and absorption of light passing through the tissue. Among various wavelengths used for optical imaging microscopy, nearinfrared (NIR) is known to have low sensitivity to such optical phenomena [5].

Therefore, nonlinear optical microscopy (NLOM), denotes for various optical imaging techniques which visualize nonlinear optical effects such as multiphoton absorption, multi-harmonic generation, is regarded very suitable for in vivo tissue for it utilizes NIR light sources (Ti:Sapphire femtosecond lasers) in general [6-9].

However, an implementation of nonlinear optical microscopy is relatively expansive than conventional optical microscopy. And is also known to very difficult because the effect of group-velocity dispersion (GVD) and setting-up the pre-chirping optics compensating the effect of GVD. Here, we introduce a relatively simple way for developing nonlinear optical microscopy with epi-illumination platform using commercial products [10].

\section{Method and Result}

The first thing to consider is the target application to get nonlinear optical images. Then, the type of microscope configuration would be selected. Among various types of microscopic configurations (e.g. upright/inverted epi-

This is an Open Access article distributed under the terms of the Creative Commons Attribution License 4.0, which permits unrestricted use, distribution, and reproduction in any medium, provided the original work is properly cited. 
illumination, or upright/inverted transmission and others), we chose type of upright epi-illumination which is suitable for in vivo imaging of live mouse. Here, one should consider whether in vivo visualization is needed or not. Actually, transmission type configuration is much suitable for ex vivo visualization of thin-sectioned tissues under $50 \mathrm{um}$. For some kinds of light generated by nonlinear effect (e.g. second harmonic generation, coherent anti-Stokes Raman spectroscopy) highly tend to proceed forward direction, only the small fraction of nonlinear light return back to their illumination path in general [11]. However, this transmission type of configuration cannot be adopted to most of in vivo imaging applications. Fortunately, the fraction of nonlinear light return back to the objective lens increases as the random scattering process in the thick tissue [12]. Therefore, if the target application is in vivo imaging, epi-illumination configuration is suitable. Else if the target application is ex vivo imaging, transmission configuration is recommended.

\subsection{Light source and Platform}

The light source is chosen to $1.4-\mathrm{W}$ femtosecond Ti:Sapphire laser (Mira 900-F, Coherent) pumped by 10W optically pumped semiconductor laser (Verdi-G10, Coherent). Compact ultrafast fiber lasers (e.g. Fidelity 1055 of Coherent, or FemtoFiber Pro of Toptica) gives lower output power but sufficient to get nonlinear images preventing tissue damage.

As a platform, we used conventional microscope (BX51, Olympus). Many microscopes including BX51 has an option to use intermediate units. And the unit named U-DP for dual port option can be set between eyepiece and objective lens turret to give access from nonlinear system to objective lens and specimen stage in the platform. This kind of configuration using conventional microscopes as platform is broadly used for their reduced efforts to generate customized platform and also their smart and familiar user interfaces [13]. Certainly, customized objective lens stage and sample stage can be used if needed. And only the part of conventional microscopes can be also used as set together with custom-made units to develop custom platform.

\subsection{Optics}

\subsubsection{Relay optics}

It is very certain that utilizing customized lens system will show higher performance than conventional products (e.g. Thorlabs, Edmund optics, Newport). However, conventional lenses give sufficient performance for nonlinear imaging for which the excitation wavelength does not exceed the range between 600-nm to 1000-nm.

During the design of relay optics using conventional optics, the first thing to consider is required tube length. That's because nonlinear optics adopts non-descan configuration for light-collection efficiency so that the collecting optics, especially cold mirror, should be inserted between the tube lens and the objective lens. And the platform used is also the major selection criteria for tube length. Therefore, tube length should be set to be longer than the sum of the space required for a cold mirror and the platform. In addition, important thing to consider is that either the exit pupil of the platform or the tube lens can be a limiting factor for the maximum fieldof-view size. Generally, commercial achromatic doublet lenses do not exceed the diameter larger than $50 \mathrm{~mm}$. Therefore, the tube length should be set between the length required for a cold mirror and the platform and the length preventing the lens system from vignetting. For our BX51 and conventional cold mirror, an achromatic doublet which has $50 \mathrm{~mm}$-diameter and $400 \mathrm{~mm}$-focal length is suitable for a tube lens.

The scan length of the relay optics is naturally set by the required magnification of the lens system. And this magnification is determined by the back aperture size of the objective lens. For using the full NA of the objective lens, it is common to set beam diameter right before the objective to $10 \%$ larger than the back aperture size, considering the beam vibration on the back aperture caused by the laser scanning. Then, the ratio between the $1.1 \mathrm{x}$ of the back aperture size and input beam size can be the magnification of the relay optics and the scan length can easily be calculate from the magnification. For a fixed objective back aperture size, the smaller magnification generally gives higher performance. That's because the larger scan angle, which makes the ray to proceed far from the central axis of the lens system so that breaks the paraxial approximation and reduces overall optical performance, is needed for achieving the same field-of-view size under the higher magnification [14].

In general, the clear aperture size of the set of scanners, especially the size of the fast axis scanner, used is the major limiting factor for input beam diameter. For instance, our system used $4-\mathrm{kHz}$ resonant scanner (CRS$4 \mathrm{k}$, Cambridge technology) which has an elliptical clear aperture about $11-\mathrm{mm}$ by $15-\mathrm{mm}$. For the longer axis of the scanner is tilted about the input axis of 45-degree, effective aperture size becomes about $9.5-\mathrm{mm}$. Therefore, we set input beam diameter of $6-\mathrm{mm}$ and the magnification of 2.5 is determined to use $20 \mathrm{X}$ objective lens with $15-\mathrm{mm}$ back aperture. The maximum possible field-of-view size of the combination of U-DP and BX51 is $500 \mathrm{um}$ by $500 \mathrm{um}$. To get this FOV size, the field angle entering the back focal point of the objective lens should be larger than 2.4-degree. Then, the required scan angle becomes to 6-degree and the scan length determined is $160-\mathrm{mm}$.

In our design process, we set the target wavelength range of $700-\mathrm{nm}$ to $900-\mathrm{nm}$ for Mira-900F, and performed computed-based simulation using Zemax software. In fact, trials using scan lenses of singleachromatic doublet did not give diffraction limited performance over the full FOV. Therefore, we designed a scan lens of dual-achromatic doublet using two identical lenses (AC508-300-B, Thorlabs) adequately separated to have effective focal length of $160-\mathrm{mm}$. On the other hand, we used one achromatic double (AC508-400-B, Thorlabs) as a tube lens. It is certain to develop the duallens tube lens would give better performance than single- 


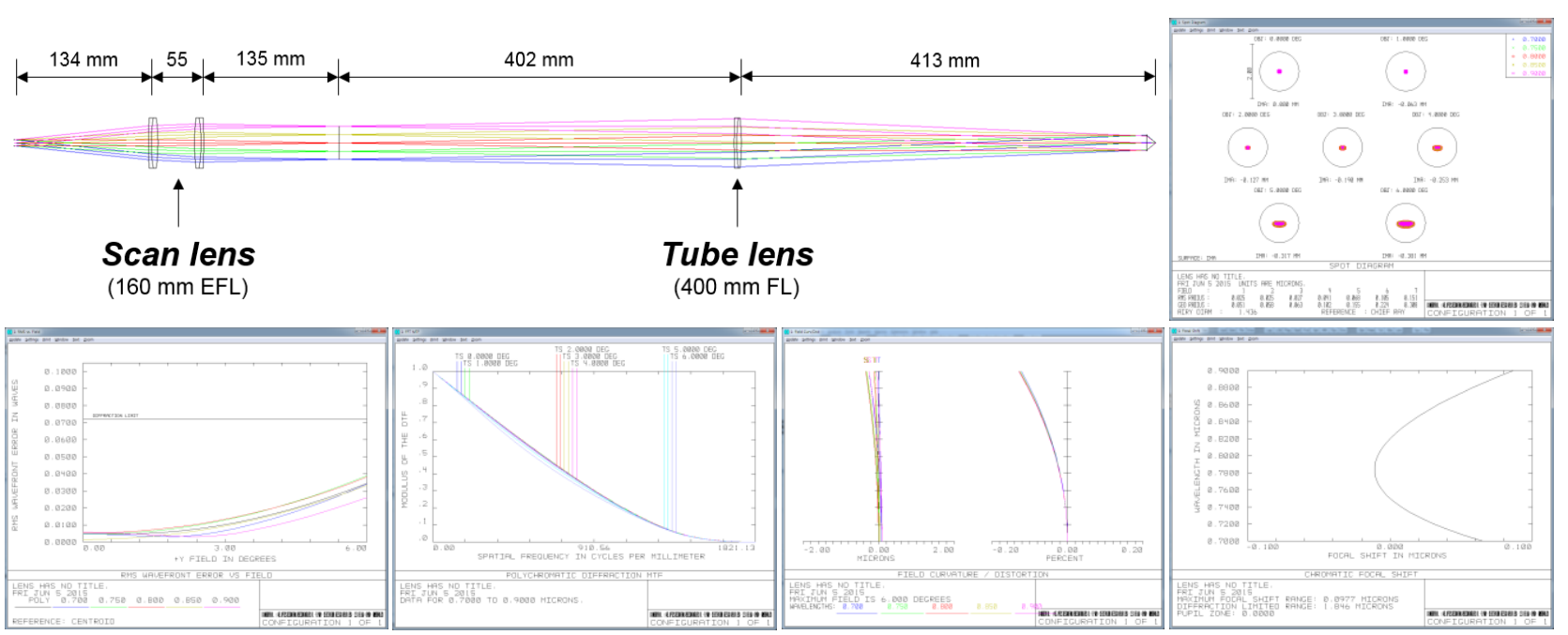

Figure 1. Design result of the relay lens. The total length of the lens system is 1137-mm including 9-mm of objective back focal length. The result showed diffraction limited performances (Spot diagram, RMS wavefront error, modulus of optical transfer functin, field curvature, distortion, and chromatic focal shift) over the full design range ; Input beam diameter $=6$-mm, tube length $>400-\mathrm{mm}$, maximum scan angle $=6$-degree, field-of-view size $=500$-um by 500 -um and the excitation wavelength between $700-\mathrm{nm}$ to $900-\mathrm{nm}$.

one, but the length available for a cold mirror and platform would be reduced by the spacing between the lenses. Figure 1 shows the design result of our relay optics. Overall optical performance satisfies the diffraction limit conditions for the entire design range.

\subsubsection{Collecting optics}

Unlike confocal epi-illumination microscopy, relay lens system designed above just acts as an illumination optics in the nonlinear system. The major difference between conventional confocal microscopy and nonlinear microscopy is the existence of descan process in detection. In confocal microscopy, emitted fluorescence or back scattered signal pass through the relay optics again and return back to the 2-axis scan system. Then, it reflected by scanners, partially or totally reflected into the photodetector by beam-splitting optics and backgroundrejected by the pinhole. This kind of detection process is called "descan". However, for nonlinear signal is so low and it has inherent optical sectioning capability without pinhole, generally filtered out before reaching the tube lens by a dichroic beam-splitter. This kind of detection process is called "Non-descan". The example of nondescan optics using commercial optical components is shown in the Figure 2 below.

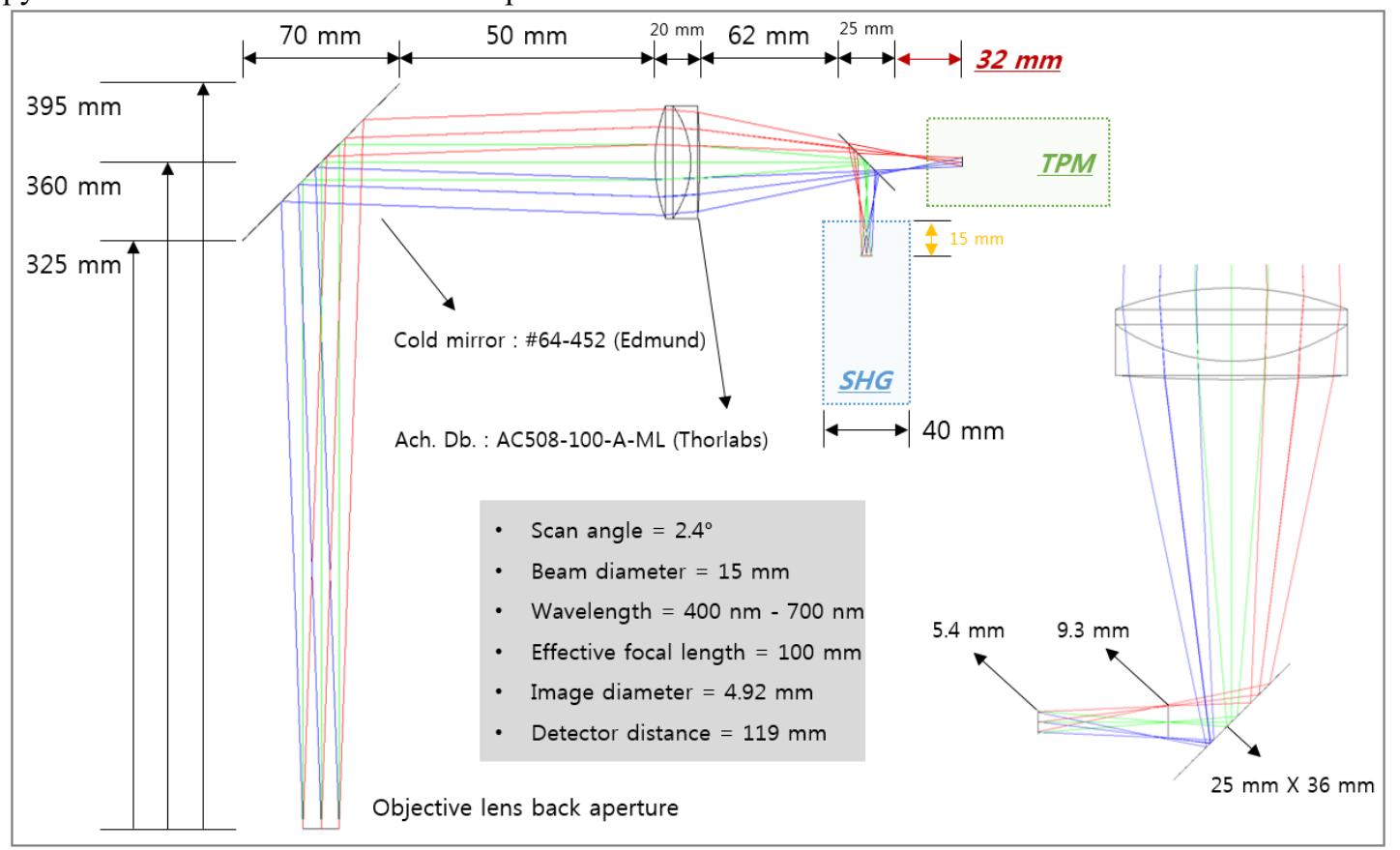

Figure 2. Design result of the collecting lens. The center of the cold mirror (\#64-452, Edmund optics) is aligned on the 360-mm beyond the back aperture of the objective lens. The cold mirror clear aperture is square and large enough to reflect most of the nonlinear signal to the collecting lens. The collecting lens is an achromatic doublet (AC508-100-A-ML, Thorlabs) which is capable of condensing nonlinear signal into the sensor area of the photomultiplier tube (Clear aperture $<10$-mm, Effective sensor area 5-mm for Hamamatsu H7422 PMT). 
The major design consideration is to collect nonlinear signal into the effective sensor area of the photodetector as much as possible. We designed the nonlinear optical system which is capable of imaging two-photon absorption effect and second harmonic generation effect. Then the collecting lens system should cover the wavelength range between $350-\mathrm{nm}$ to $650-\mathrm{nm}$ to fully collect the back scattered second harmonic signal and the emitted two-photon absorption signal. Only for the case of which the cold mirror position is far from the objective lens, the clear aperture of the cold mirror should be considered to collect nonlinear signal gathered through large field angle.

\subsubsection{Pre-chirping optics}

The group-delay dispersion (GDD) of the optical components can be calculated theoretically by multiplying their thickness to theoretical group-delay dispersion (GVD) which is specified in glass catalogues. And the total GDD of the optical lens system is simply the numerical sum of GDD values of each components. For our relay system designed above, total GDD is $2753.3-\mathrm{fs}^{2}$ for $800-\mathrm{nm}$ excitation. And we designed the pre-chirping optics which is capable of compensating $6000-\mathrm{fs}^{2}$ of GDD including the GVD caused by the objective lens using a pair of SF10 prisms. The detailed designed procedure is well-explained in the paper written by S. Yang et al [10].

\section{Conclusion}

In fact, there are various different configurations realizing nonlinear optical microscopy. Even wide-field nonlinear microscopy has been realized [15]. And various technique has shown to enhance nonlinear signal detected. Our purpose here is to show simplified technique to build nonlinear optical system. In this paper, dual modal (Twophoton absorption microscopy and second harmonic generation microscopy) epi-illumination type imaging system was designed by using only four commercial achromatic doublet lenses and one pair of SF10 prisms. Computer-based simulation shows the design result has sufficient optical performance to develop nonlinear microscopy. Further developing the system into threemode, including confocal laser scanning microscopy, by inserting the descanned confocal optics composed of a condensing lens and a pinhole is also possible. For a future work, we are going to measure the actual imaging performance of the developed system experimentally.

\section{References}

1. J. W. Lichtman and J.-A. Conchello, "Fluorescence microscopy.," Nat. Methods 2, 910-919 (2005).

2. M. Minsky, "Microscopy Apparatus," US Pat. 3013467 3013467, 5 (1961).

3. J.-A. Conchello and J. W. Lichtman, "Optical sectioning microscopy.," Nat. Methods 2, 920-931 (2005).
4. M. Rajadhyaksha, R. R. Anderson, and R. H. Webb, "Video-rate confocal scanning laser microscope for imaging human tissues in vivo.," Appl. Opt. 38, 2105-2115 (1999).

5. R. Weissleder, "A clearer vision for in vivo imaging Progress continues in the development of smaller, more penetrable probes for biological imaging . Toward the phosphoproteome," Nat. Biotechnol. 19, 316-317 (2001).

6. T. T. Le, I. M. Langohr, M. J. Locker, M. Sturek, and J.-X. Cheng, "Label-free molecular imaging of atherosclerotic lesions using multimodal nonlinear optical microscopy," J. Biomed. Opt. 12, 054007 (2007).

7. P. J. Campagnola and L. M. Loew, "Secondharmonic imaging microscopy for visualizing biomolecular arrays in cells, tissues and organisms.," Nat. Biotechnol. 21, 1356-1360 (2003).

8. B. Masters and P. So, "Confocal microscopy and multi-photon excitation microscopy of human skin in vivo.," Opt. Express 8, 2-10 (2001).

9. W. L. Chen, P. S. Hu, A. Ghazaryan, S. J. Chen, T. H. Tsai, and C. Y. Dong, "Quantitative analysis of multiphoton excitation autofluorescence and second harmonic generation imaging for medical diagnosis," Comput. Med. Imaging Graph. 36, 519-526 (2012).

10. S. Yang, K. Lee, Z. Xu, X. Zhang, and X. Xu, "An accurate method to calculate the negative dispersion generated by prism pairs," Opt. Lasers Eng. 36, 381387 (2001).

11. L. Moreaux, O. Sandre, and J. Mertz, "Membrane imaging by second-harmonic generation microscopy," J. Opt. Soc. Am. B 17, 1685 (2000).

12. C. L. Evans, E. O. Potma, M. Puoris'haag, D. Côté, C. P. Lin, and X. S. Xie, "Chemical imaging of tissue in vivo with video-rate coherent anti-Stokes Raman scattering microscopy.," Proc. Natl. Acad. Sci. U. S. A. 102, 16807-16812 (2005).

13. I. Veilleux, J. A. Spencer, D. P. Biss, D. Cote, and C. P. Lin, "In Vivo Cell Tracking With Video Rate Multimodality Laser Scanning Microscopy," IEEE J. Sel. Top. Quantum Electron. 14, 10-18 (2008).

14. A. Negrean and H. D. Mansvelder, "Optimal lens design and use in laser-scanning microscopy," Biomed. Opt. Express 5, 1588 (2014).

15. D. Oron, E. Tal, and Y. Silberberg, "Scanningless depth-resolved microscopy.," Opt. Express 13, 14681476 (2005). 